\title{
Invited review: Genome-wide association analysis for quantitative traits in livestock - a selective review of statistical models and experimental designs
}

\author{
Markus Schmid and Jörn Bennewitz \\ University Hohenheim, Institute of Animal Science, Garbenstrasse 17, 70599 Stuttgart, Germany \\ Correspondence to: Jörn Bennewitz (j.bennewitz@uni-hohenheim.de)
}

Received: 23 June 2017 - Revised: 21 July 2017 - Accepted: 10 August 2017 - Published: 29 September 2017

\begin{abstract}
Quantitative or complex traits are controlled by many genes and environmental factors. Most traits in livestock breeding are quantitative traits. Mapping genes and causative mutations generating the genetic variance of these traits is still a very active area of research in livestock genetics. Since genome-wide and dense SNP panels are available for most livestock species, genome-wide association studies (GWASs) have become the method of choice in mapping experiments. Different statistical models are used for GWASs. We will review the frequently used single-marker models and additionally describe Bayesian multi-marker models. The importance of nonadditive genetic and genotype-by-environment effects along with GWAS methods to detect them will be briefly discussed. Different mapping populations are used and will also be reviewed. Whenever possible, our own real-data examples are included to illustrate the reviewed methods and designs. Future research directions including post-GWAS strategies are outlined.
\end{abstract}

\section{Introduction}

Quantitative or complex traits are controlled by many genes and environmental factors. Most traits in livestock breeding are quantitative traits, and there is a tremendous interest in analyzing these traits, e.g., with the aim to estimate breeding values of selection candidates or to map the underlying genes or chromosomal regions (quantitative trait loci, QTL). In earlier QTL mapping studies sparse genetic marker maps and linkage analysis were used to map QTL in experimental populations like F2 crosses or half-sib designs (e.g., Weller et al., 1990). Although many QTL were mapped, the mapping precision was usually low and only in a few exceptional cases was the underlying gene identified.

With the advent of high-density SNP arrays for most of the livestock species, it became possible to apply genomewide association studies (GWASs). The underlying principle of GWASs is to test the SNPs for trait associations. The interpretation of statistically significant SNP trait associations is that the SNP is in linkage disequilibrium (LD) with a causative gene and that gene and the SNP are tightly linked.
The latter is the case because the level of LD is a function of the distance between two loci on the chromosome.

One of the main reasons for mapping QTL was to use mapped QTL for selection purposes in marker-assisted selection schemes (Dekkers, 2004). However, the success of these selection schemes was only very limited, mainly because the explained variance by the mapped QTL was very small. In order to overcome these limitations, Meuwissen et al. (2001) transferred marker-assisted selection on a genome-wide scale and developed statistical models to estimated genomic breeding values that rely on genome-wide and dense marker data but not on results from mapping experiments. The selection of breeding candidates based on genomic estimated breeding values became known as genomic selection, and it is implemented in many livestock genetic breeding programs, where it accelerates genetic gain substantially.

Despite the success of genomic selection, mapping genes for complex traits is still a burning issue in livestock genetics. Goddard et al. (2016) listed three main reasons for this. The first is to improve genomic selection. Second, GWAS results can increase biological knowledge about trait expression. The function of GWAS-identified genes can be used to 
derive and validate hypotheses about trait synthesis. This is of special interest for novel traits that eventually will be included in the selection goal or that might be controlled by tailored drugs or feeding strategies, like feather pecking in laying hens, greenhouse gas emission in ruminants or nutrient efficiency. Third, GWAS results can provide information on the genetic architecture of the quantitative trait; i.e., we may be interested in how many genes control the genetic variance, what the effect sizes are, how important nonadditive genetic effects are and so on.

Different statistical methods and types of populations have been used in livestock GWAS experiments. In this study, we will review the most commonly used methods and mapping populations. First, single- and multi-marker GWAS models are presented. Next, we describe the importance of nonadditive genetic and genotype-by-environment effects and show how these can be modeled in GWASs. Different mapping populations are used and these will be described in the following section. This review ends with a discussion, where future research directions including post-GWAS strategies are outlined. Whenever possible, our own real-data examples are included to illustrate the reviewed methods and designs. Because GWASs rely on LD between SNPs and causative genes, we start with a brief description of the most commonly used LD measure and its expectation.

\section{Linkage disequilibrium measure $r^{2}$ and its expectation}

Assume two loci, $A$ and $B$, with two alleles each, i.e., alleles $A$ and $a$ and alleles $B$ and $b$, with allele frequencies $f_{A}, f_{a}, f_{b}$ and $f_{b}$. The haplotype frequencies of the haplotypes $A B, A b, a B$ and $a b$ are denoted by $f_{A B}, f_{A b}, f_{a B}$ and $f_{a b}$, respectively. Following Hill and Weir (1994) the LD between these loci can be calculated as $r^{2}=\frac{\left(f_{A B} f_{a b}-f_{A b} f_{A b}\right)^{2}}{f_{A} f_{a} f_{B} f_{b}}$. This measure has some convenient properties. It is bounded between 0 and 1, with 1 being a perfect LD. Assume locus $A$ is a gene and $B$ is an SNP used in a GWAS. In this case, the fraction of gene variance explained by the SNP is $r^{2}$ (although the LD and the gene variance remains unknown until the causative gene itself is identified). The expectation of $r^{2}$ can be expressed as $E\left(r^{2}\right)=1 /\left(1+4 N_{\mathrm{e}} c\right)$, with $N_{\mathrm{e}}$ being the effective population size, and $c$ denotes the recombination rate between the two loci (Sved, 2009; Tenesa et al., 2007). From this expression, it becomes obvious that the expected LD decays fast with increasing distances between loci, especially if the effective population size is large. The following example illustrates this. Stratz et al. (2014) investigated the LD structure of a segregating Piétrain pig population. They used SNP chip genotypes (porcine 60K BeadChip, Illumina Inc., San Diego, CA) of nearly 900 Piétrain boars for the $\mathrm{LD} r^{2}$ calculation for SNP pairs with a maximum distance of 5 megabases $(\mathrm{Mb})$. The results are shown for Sus scrofa chromosome 1 (SSC 1) in Fig. 1 as a histogram of
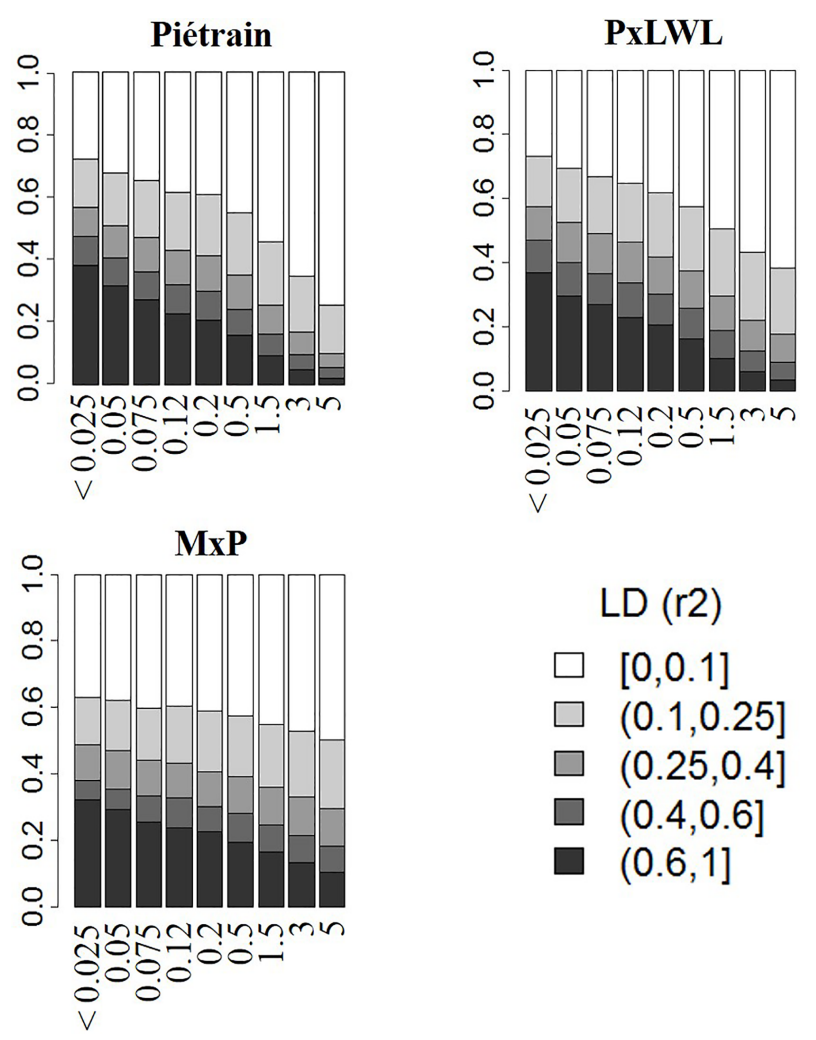

Figure 1. Linkage disequilibrium decay as a function of the marker distance in a purebred Piétrain population and in F2 crosses derived from closely (Piétrain $\times$ Landrace/Large White) and distantly (Piétrain $\times$ Meishan) related founder breeds (Sus scrofa chromosome $1)$.

mean $r^{2}$ for bins of SNP pair distances. The level of LD decreases strongly for larger distances. Compared to humans, long-range LD blocks are more common in livestock, especially in dairy cattle. This is due to the intensive use of relatively few sires for breeding the next generation, which results in a relatively small effective population size.

\section{Single-marker models}

Single-marker GWASs fit one SNP at a time, usually in a mixed linear model (Yang et al., 2014). When assuming a single SNP $j$ with genotypes coded as the number of copies of the allele with the minor frequency at the SNP for each individual $i\left(x_{i j}=0,1\right.$ or 2$)$, the following model is frequently used:

$y_{i}=\mu+b_{j} x_{i j}+u_{i}+e_{i}$.

Thereby, $y_{i}$ is the trait record of individual $i, \mu$ denotes the fixed mean (assuming no other fixed effects exist) and $b_{j}$ is the regression coefficient for SNP $j$ to be estimated. In this parametrization, the SNP effect represents the gene substitution effect (Falconer and Mackay, 1996). The term $e_{i}$ de- 
notes the random residual and $u_{i}$ the random polygenic effect of the individual. The distributional assumption of the polygenic effects is $u \sim N\left(0, A \sigma_{u}^{2}\right)$, with $A$ being the relationship matrix either to be estimated from pedigree or from SNP data and $\sigma_{u}^{2}$ being the polygenic variance component. The test for trait association is done by testing $b_{j}$, being different from 0 , which results in an error probability or $p$ value. In a GWAS, one SNP at a time is fitted to the model, resulting in multiple tests. In order to correct for these multiple tests, several approaches can be applied. The most common ones are the Bonferroni correction and the false discovery rate (FDR). Often, the Bonferroni correction is used to determine genome-wide significance thresholds and the FDR to assess how many of the associations reaching the significance level are false positives. The level of multiple testing can be enormous, especially if dense SNP chips or sequence data are used, and these SNPs are in LD and thus do not segregate independently. In these common situations, the Bonferroni correction is very stringent, and thus the results are conservative. More details about corrections for multiple testing in QTL mapping can be found in Fernando et al. (2004).

The polygenic component in Eq. (1) is important to capture population stratification effects and thus to prevent an inflation of type-I errors (e.g., MacLeod et al., 2010). Unlike in plant breeding, it is very convenient that for many livestock mapping populations the pedigree is known, and hence the relationship matrix needed to model this component adequately can be calculated using this information. If this is not possible, genetic markers can be used to set up a genomic relationship matrix (GRM) (VanRaden, 2008). If GRMs are used, the question is whether the SNP to be tested for association (or indeed the SNPs being in LD with this SNP) should also be used to set up the GRM or not. In the case of an inclusion, the SNP appears twice in the model and is treated once as a fixed and once as a random effect. Consequently, the SNP has to compete against itself, which seems somewhat counterintuitive. Indeed, Yang et al. (2014) showed that this results in a reduced mapping power. These authors recommended the exclusion of all SNPs that are located on the same chromosome as the SNP to be tested from the GRM. However, a recent article by Gianola et al. (2016) on GWASs with a GRM suggests that double-fitting the SNP effects (as fixed and random effects) is a less severe problem than previously thought. Another way of modeling population structure is to fit principal components (Patterson et al., 2006), but, as Hayes (2013) pointed out, it is not exactly traceable which variation source they actually remove. It may be noted that removing population structure effects is not straightforward when generalized linear models (e.g., Poisson models) are applied (Lutz et al., 2017).

In a recent study we conducted a single-marker mixed model GWAS in Holstein dairy cattle data (Streit et al., 2013a). In brief, there were around 2300 progeny-tested bulls available, which were genotyped with the bovine $50 \mathrm{~K}$ BeadChip (Illumina Inc., San Diego, CA). Qanbari et al. (2010) investigated the LD structure in this population. The trait considered was protein milk yield, and the relationship matrix of the bulls was established using pedigree information. The data set was split into a discovery data set (about 1800 bulls) for GWASs and a validation data set (500 bulls). The latter was exploited to confirm significant SNP associations identified in the discovery data set. FDR was applied to account for multiple testing. The results are shown in a so-called Manhattan plot in Fig. 2, with the negative decadic logarithm of the $p$ value for each SNP on the $y$ axis and the chromosomal position on the $x$ axis (a common way of presenting GWAS results). Overall, 450 significant SNPs were identified with an FDR of maximally $7 \%$. Of these, 69 associations were also significant in the validation data set. Hence, these associations could be confirmed in the same population. Some of the identified trait-associated SNP clusters are located closely to well-known candidate genes segregating in the population (e.g., DGATl on Bos taurus autosome (BTA) 14).

\section{Bayes multi-marker models}

As stated above, the level of multiple testing can be enormous with dense SNP data, and stringent thresholds are needed in order to prevent an inflation of type-I errors. In addition, it is possible that the effect of a gene is only in part captured by a single marker due to imperfect LD but might be better explained jointly by the SNPs surrounding the gene. In order to overcome these limitations, multi-marker models that fit all SNPs simultaneously as random effects in the model were introduced for GWASs. Such models are able to deal with the problem that the number of SNPs often exceeds the number of observations. A general form of the model is as follows:

$$
y_{i}=\mu+\sum_{j=1}^{n-\mathrm{SNP}} b_{j} x_{i j}+e_{i} .
$$

Compared to Eq. (1), the main difference is that all SNPs are fitted simultaneously as random effects These models were originally developed for genomic selection purposes (Meuwissen et al., 2001) but have been shown to be very useful also for GWASs (Sahana et al., 2011; Goddard et al., 2016). The distributional assumptions of the SNP effects differ from model to model. The SNP-BLUP (Best Linear Unbiased Prediction) model assumes that all SNP effects come from one normal distribution with a small variance. This implies that the trait genetic variance is more or less equally distributed over the genome. This is a strong assumption and probably unrealistic for many quantitative traits. For this reason, Meuwissen et al. (2001) proposed two Bayesian models. The method called BayesA assumes a $t$ distribution of the SNP effects, which is thicker-tailed compared to the normal distribution, depending on the degrees of freedom. BayesB models assume that only a fraction of the SNPs $(\pi)$ has an 


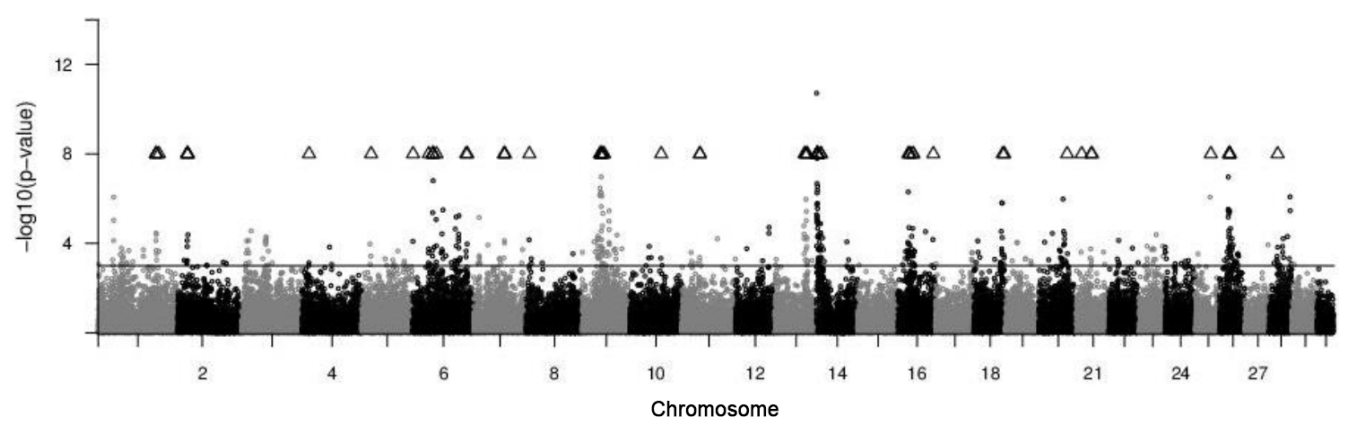

Figure 2. Test statistics of a single-marker GWAS for the trait milk protein yield in a sample of the German Holstein population. The solid line corresponds to a significance level of $P=0.001$. Significant SNPs are indicated by triangles (taken from Streit et al., 2013a).

effect on the variance of a trait. For this fraction, a $t$ distribution is assumed. Since the landmark paper by Meuwissen et al. (2001), further Bayes models were introduced (reviewed by Gianola, 2013). Verbyla et al. (2009) and Verbyla et al. (2010) proposed Bayesian stochastic search variable selection, which was also named BayesC by these authors. This model assumes two $t$ distributions: one with a large variance for the $\pi$ SNP fraction and one with a small variance for the $1 \pi$ fraction (e.g., 100 times smaller). SNPs belonging to the latter fraction hardly contribute to the genetic variance of a trait (or do not do so at all), and their effects are close to 0 . The assumptions of BayesR, introduced by Erbe et al. (2012), are based on a mixture of normal distributions for the SNP effects.

Inference about an SNP trait association can either be drawn by the effect of a single SNP or by the posterior probability that the SNP effect comes from a distribution with large variance (in BayesB, C and $\mathrm{R}$ ). The SNP effect is a random effect and a marginal effect, i.e., an effect corrected for all other SNP effects. This effect is sometimes also denoted as a conditional marker effect because the effects are drawn from conditional posterior distributions. The marginal marker effect is different from the effect obtained in Eq. (1) and, indeed, very sensitive to the SNP density. With increasing SNP density, the level of shrinkage towards 0 becomes stronger. Thus, it seems more straightforward to draw an inference by considering SNP effects within a window of defined size (e.g., 1 centimorgan $(\mathrm{cM})$ ) jointly and estimate the window genetic variance. Fernando et al. (2017) used the window genetic variance to calculate the window posterior probability of association (WPPA). This criterion has some nice properties. If a WPPA threshold of, e.g., 0.95 is used to declare an association as plausible, this results in a proportion of false positives of 0.05 . This holds true if the data-generating model and the data-analysis models are similar. The WPPA criterion is convenient to compute, does not suffer from increasing marker density and produces an association criterion that is directly interpretable as the probability of window trait association.
For genomic predictions, the Bayesian methods often outperformed the SNP-BLUP model in computer simulations (e.g., Meuwissen et al., 2001), but this was often not the case in real data. This is probably due to the fact that many genes affect a trait and due to the long-range LD in livestock breeds, which results in many SNPs being in LD with a gene. However, this equal performance of the models does not hold for their use in GWASs. We used the Holstein dairy cattle data set mentioned above (Streit et al., 2013a) to compare the models SNP-BLUP, BayesA and BayesC in a GWAS for milk protein yield. In the BayesA and BayesC models, $t$ distributions with 4 degrees of freedom (df) were assumed. The fraction of SNPs coming from the distribution with the large variance was $\pi=0.2$. In BayesC, the variance of this distribution was 100 times larger than the variance of the a priori $1 \pi$ fraction of SNP effects. Gibbs sampling was used to draw samples from the posterior distributions using the program BayesDsamples (Wellmann and Bennewitz, 2012). The SNP effect estimates were used to calculate window genomic breeding values for windows of five consecutive SNPs $\left(\mathrm{GEBV}_{\mathrm{W}}\right)$ using standard notations (Falconer and Mackay 2007; Bennewitz et al., 2017). From these, the expected $\mathrm{GEBV}_{\mathrm{W}}\left(\mathrm{E}\left(\mathrm{GEBV}_{\mathrm{W}}\right)\right)$ was subtracted in order to pinpoint trait-associated chromosomal regions. The $\mathrm{E}\left(\mathrm{GEBV}_{\mathrm{W}}\right)$ was calculated under the assumption of an equal distribution of the additive genetic variance across the genome; i.e., it was assumed that all genomic regions contribute equally to the additive genetic variance (for further details, see Bennewitz et al., 2017, Appendix). A putative QTL was assumed in those windows that showed a deviation greater than 0 , i.e., $G E B V_{W}-E\left(G E B V_{W}\right)>0$. The plot of the $G B V_{W}$ deviations are shown in Fig. 3 for all three methods. When applying SNP-BLUP, only the window surrounding DGAT1 on BTA 14 showed evidence for trait association. BayesA produced around 10 additional and BayesC around 30 additional signals. The results are shown for BTA 6 in detail in Fig. 4, for which the single-marker GWAS (Eq. 1) revealed a confirmed trait-associated region (Fig. 2). BayesC clearly produced two signals on this chromosome, which were not detected by the two other methods. Following this, it seems that 
the Bayes methods, especially BayesC, are much more able to zoom into the genome and to pinpoint causative genomic regions. BayesR, which used a mixture of normal distributions with four components, was not investigated in this study but was propagated as a suitable method for the GWAS by Goddard et al. (2016).

Compared to single-marker GWASs, the application of these multi-marker methods is not straightforward and needs some carefully chosen parameters. For SNP effect estimation, the most important ones are the Markov chain Monte Carlo (MCMC) length, $\pi$, the variance scaling factor and the degrees of freedom. To our best knowledge, the length of the MCMC suitable for GWASs has not been sufficiently investigated until now. A small number of df results in a heavytailed $t$ distribution and only large-effect SNPs will be identified (small effects will be regressed back to 0). Consequently, the number of false positives might be small but this compromises the power. The opposite holds true for larger numbers of df. The size of the windows for inference purposes, e.g., by the WPPA criterion, affects the power additionally. Larger window sizes result in an increased power but also in a reduced precision, i.e., the size of a trait-associated genomic region is larger (Bennewitz et al., 2017). There is a trade-off between power and precision. An obvious solution would be to start with larger window sizes, e.g., of $1 \mathrm{cM}$, to find significant trait-associated chromosomal regions and subsequently to reduce the windows size to fine-map the region.

We further tested nonparametric additive regression models originally adopted for genomic selection (Bennewitz et al., 2009) for GWASs using this data set. In contrast to Bayesian methods, no prior information is needed. However, this method did not produce very clear GWAS signals, which was similar to the SNP-BLUP model (not shown).

\section{Nonadditive genetic and interaction effects}

\subsection{Dominance and imprinting}

The most important nonadditive genetic effects are dominance and epistasis (Falconer and Mackay, 1996). It is well known that additive genetic variance is most important, and compared to this, dominance and epistatic variances are in general much smaller in size (Hill et al., 2008). However, this does not mean that there are no dominance effects of a detectable size (Wellmann and Bennewitz, 2011). Recent SNP-based investigations revealed that dominance variance can be substantial (e.g., Ertl et al., 2014; Su et al., 2012). Bolormaa et al. (2015) used a large-scale experiment with about 10000 cattle, which were phenotyped for 16 quantitative traits and genotyped with dense SNP panels. They conducted a GWAS using single-marker regression analysis and found many trait-associated SNPs with a dominance effect. Moreover, the estimates of the dominance variance as a proportion of the phenotypic variance across the traits was between 0 and $42 \%$ with a median of $5 \%$. Hence, it seems
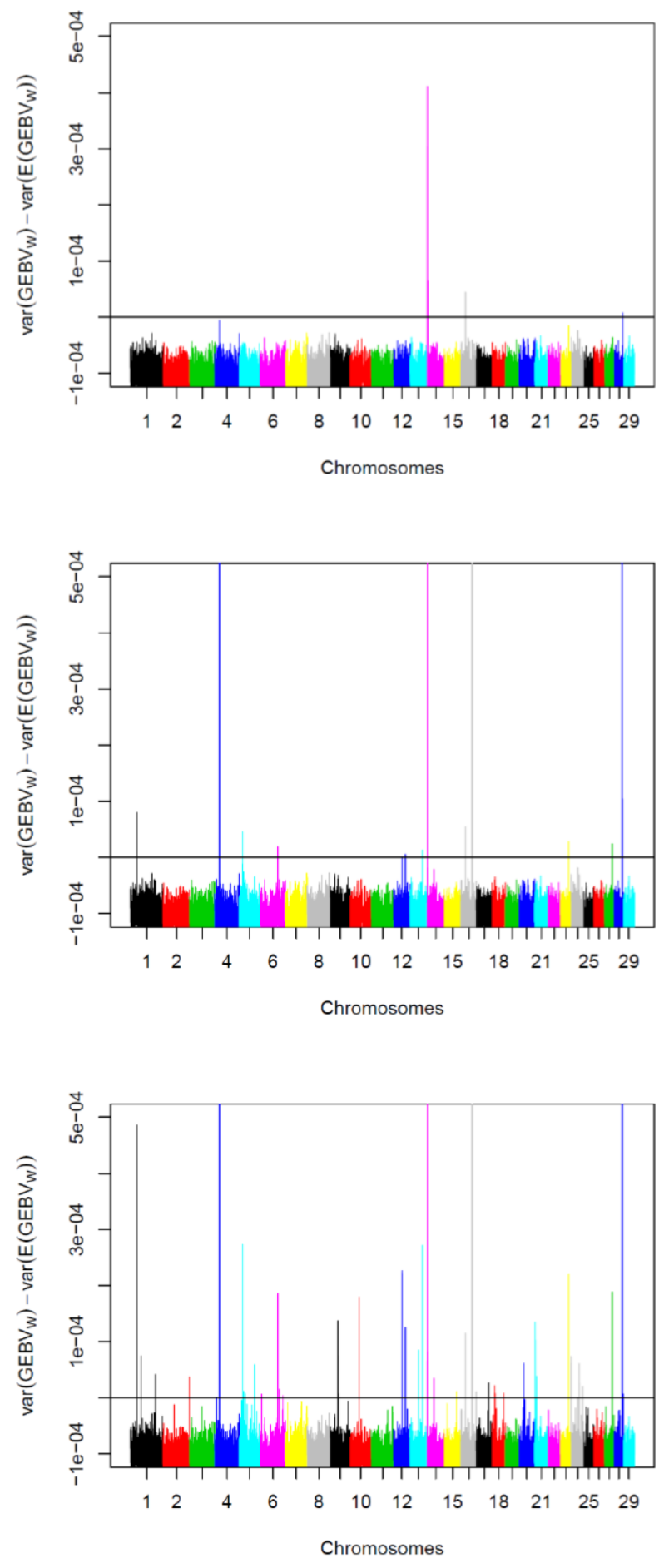

Figure 3. Results of a window-based multi-marker GWASs in a sample of the German Holstein population using the models SNPBLUP (top panel), BayesA (middle panel) and BayesC (bottom panel). For each window, the deviation of the variance of the genomic estimated breeding value from its expected value is shown. The solid line corresponds to a deviation of 0 . 
that dominance is an important source of genetic variation for some traits, and it seems appropriate to use this additional variation if the data structure permits it (i.e., genotypes and phenotypes are collected from the same individual). For example, the data set of Streit et al. (2013a) used in the previous section does not allow for dominance effect estimation because daughter yield deviations were used.

Single-marker association models (Eq. 1) can be extended straightforwardly towards modeling dominance. In addition to the regression on the SNP gene content, a regression on a heterozygous indicator variable is included, which represents the dominance deviation effect (Falconer and Mackay, 1996). Because dominance is modeled explicitly, the regression coefficient on the gene content no longer represents the gene substitution effect but the additive gene effect. This parametrization invokes one additional parameter to be estimated. Wellmann and Bennewitz (2011) showed that dominance and additive effects are dependent on each other in a complicated manner. Large dominance effects are usually observed for genes with large additive effects, which means that overdominance is a rare event. Therefore, in singlemarker GWAS, a two-step procedure is often applied. In step one, only additive effects are fitted to the model. In the second step, dominance is included, and this extended model is applied only to SNPs with significant additive effects. This way of modeling dominance in single-marker GWAS models was chosen by, e.g., Bolormaa et al. (2015).

The BayesC model was extended towards accounting for dominance, resulting in BayesD (Wellmann and Bennewitz, 2012). This model uses priors for the additive and dominance effects and the gene frequencies that resemble the complicated relationship between them. Roughly speaking, for small additive effects, the dominance deviations fluctuate around 0 . With increasing additive effect sizes, the dominance deviation becomes larger and points in general to the homozygous genotype associated with the larger phenotypic value. The sign of additive and dominance effects depend on the gene frequency. Following this, it is unlikely that the contribution of the gene to the overall genetic variance is large. The latter is assumed because selection shifts the gene frequency towards a value where the variance contribution is small. Details can be found in Wellmann and Bennewitz (2011). In a recent study, we compared BayesC and BayesD for GWASs using simulated and real data sets (Bennewitz et al., 2017). We used the WPPA criterion for inference purposes and found a shift in power that was between -2 and $9 \%$. Dominance is an interaction effect of the two alleles at a locus. Their effects are captured in the association analysis by matched haplotype pairs, i.e., diplotypes. Diplotypes show a faster decay around a focal point in the genome compared to haplotypes. Hence, it can be expected that BayesD improves the mapping precision as well, but this needs higher marker densities.

Imprinting seems to be a non-negligible source of variation for some quantitative traits in livestock. Trait-associated
SNPs with imprinting effects can be detected by linkage analysis and GWASs. Models to do such analyses are presented in Mantey et al. (2005) and Hu et al. (2015).

\subsection{Epistasis}

The statistical interaction between SNPs is termed epistasis. The role of epistasis in the manifestation of quantitative traits has been subject to some debate during the last decades (e.g., Carlborg and Haley, 2004; Hill et al., 2008, among others). Detecting pairwise epistatic trait-associated SNPs can be done in principle by extending Eq. (1) by a second SNP and interaction terms between them. Even in this simple form of epistasis, i.e., pairwise epistasis, the model becomes much more complex because four interaction terms have to be fitted (additive-by-additive, additiveby-dominance, dominance-by-additive and dominance-bydominance). In addition, the search for epistatic effects involves expanding from one dimension genome screenings (as for additive effects) towards two or even higher dimensions. This requires many statistical tests and thus increases the problem of multiple testing enormously. Therefore, in addition to the need of dense SNP maps, a large sample size is needed in order to obtain a sufficient power to detect epistatic effects. It is sometimes argued that SNPs involved in epistasis also show additive effects. Based on this assumption, epistatic interactions are sometimes fitted only for SNPs that were significant in a previous GWAS run without fitting epistasis. This reduces the number of tests dramatically. Wei et al. (2014) reviewed statistical models to detect epistasis by GWASs.

\subsection{Genotype-by-environment interaction}

Genotype-by-environment interactions $(\mathrm{G} \times \mathrm{E})$ are defined as the difference between genotype effects measured in different environments. A recent review of $\mathrm{G} \times \mathrm{E}$ in livestock can be found in Hayes et al. (2016). G $\times E$ can result in re-ranking effects; i.e., one genotype is superior in one environment, but inferior in the other environment. $\mathrm{G} \times \mathrm{E}$ scaling effects refer to the same ranking of genotypes, but the differences are larger in one environment compared to another environment. In general, two statistical methods are applied to test for $\mathrm{G} \times \mathrm{E}$. Multiple-trait models treat the phenotypic records of a trait collected in different environments as different traits and calculate a genetic correlation between them. A deviation of this correlation from 1 (e.g., < 0.8 ) can be interpreted as evidence for $\mathrm{G} \times \mathrm{E}$. In reaction norm models, the environment is described by a continuously distributed environmental descriptor and the phenotype is modeled as a function of the environment, where the phenotype is produced. Typical environmental descriptors are temperature-humidity indices (Hayes et al., 2003), average herd production levels as an indicator of the feeding level (Calus et al., 2002; Hayes et al., 2003) or herd disease levels (e.g., somatic cells score as an 


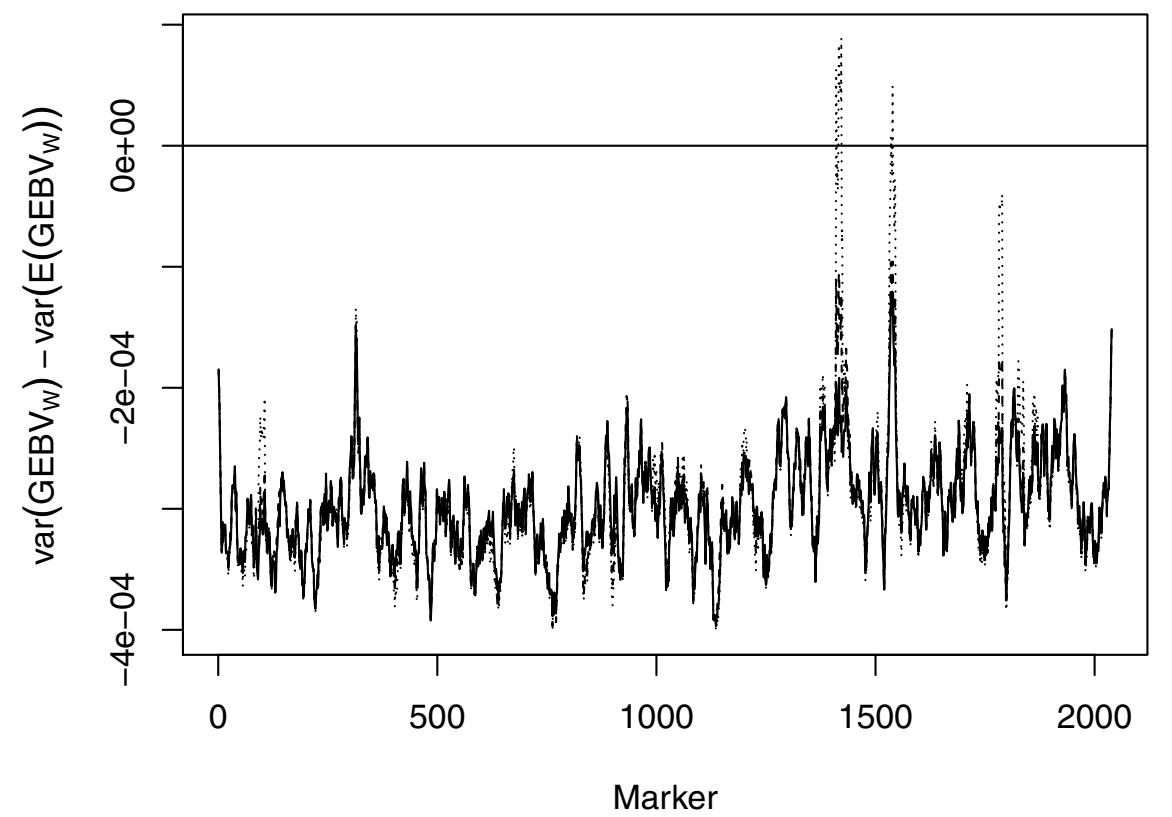

Figure 4. Comparison of GWAS results generated with SNP-BLUP (solid line), BayesA (dashed line) and BayesC (dotted line) on BTA 6 in a sample of the German Holstein population. For each window, the deviation of the variance of the estimated genomic breeding value from its expected value is shown. The horizontal solid line corresponds to a deviation of 0 .

indicator of udder health and infection pressure on the farm; Streit et al., 2013b). Hayes et al. (2009) proposed a two-step reaction norm GWAS model to identify SNPs that showed $\mathrm{G} \times \mathrm{E}$ effects. In the first step, a random regression reaction norm model is applied to sires with sufficient progeny information in different environments as follows:

$y_{i j k}=\mu+\sum_{m=0}^{1} s_{j m} \cdot E_{k}^{m}+e_{i j k}$.

Hereby, $y_{i j k}$ is the observation of offspring $i$ of sire $j$ recorded in herd $k$ with average level of the environmental descriptor $E_{k}, s_{j m}$ is the random sire effect of sire $j$ of order $m$ and $e$ denotes the residual. The covariance structure of the sire regression coefficients is

$\operatorname{var}\left[\begin{array}{c}s_{0} \\ s_{1}\end{array}\right]=A \otimes\left[\begin{array}{cc}\sigma_{s_{0}}^{2} & \sigma_{s_{0} s_{1}}^{2} \\ \sigma_{s_{0} s_{1}}^{2} & \sigma_{s_{1}}^{2}\end{array}\right]$.

Note that this is a sire model. The residuals contain about three-quarters of the genetic variance. Thus, if $\mathrm{G} \times \mathrm{E}$ is present, the residuals are heterogeneous, and this should be modeled as well. This model estimates two sire effects: one for the slope and one for the intercept of the reaction norm. If the mean of the environmental descriptor is set to 0 , the intercept solutions of the sire regression coefficients are sire estimates for the general production level, i.e., the production level in the average environment. The sire's reaction norm slope effects represent the environmental sensitivity of the sire. In the second step, the sire's intercept and slope solutions are used as observations in a GWAS model, e.g., Eq. (1). GWAS hits for the slope identify environmentally sensitive trait-associated SNPs and thus SNPs involved in $\mathrm{G} \times \mathrm{E}$. Equation (3), shown above, is a random regression model. As a result, the sire solutions for intercept and slope are regressed back to 0 , which might compromise the power for a subsequent GWAS. Alternatively a fixed regression could be applied (known as the Finlay-Wilkinson regression in plant breeding), but the behavior of such a model for GWAS purposes needs to be investigated in detail.

In an earlier study, we used the two-step approach described above to map G $\times$ E SNPs in German Holsteins (Streit et al., 2013a). We used milk production test-day records of around 1.3 million daughters sired by 2300 sires with 12 million first lactation test records. We applied a two-step procedure to map SNPs associated with protein production $\mathrm{G} \times \mathrm{E}$. Initially, a reaction norm random regression model (Eq. 3) was applied to the data, and subsequently we used the slope sire solutions as observations in a single-marker association model (Eq. 1). The results are shown in Fig. 5. We detected 351 significant trait-associated G $\times$ E SNPs, of which 44 could be confirmed in the same population. Generally, the results are very similar to those of the general milk protein production (see Fig. 2). Indeed, many trait-associated SNPs were also involved in $\mathrm{G} \times \mathrm{E}$. This is discussed in detail in Streit et al. (2013a). 


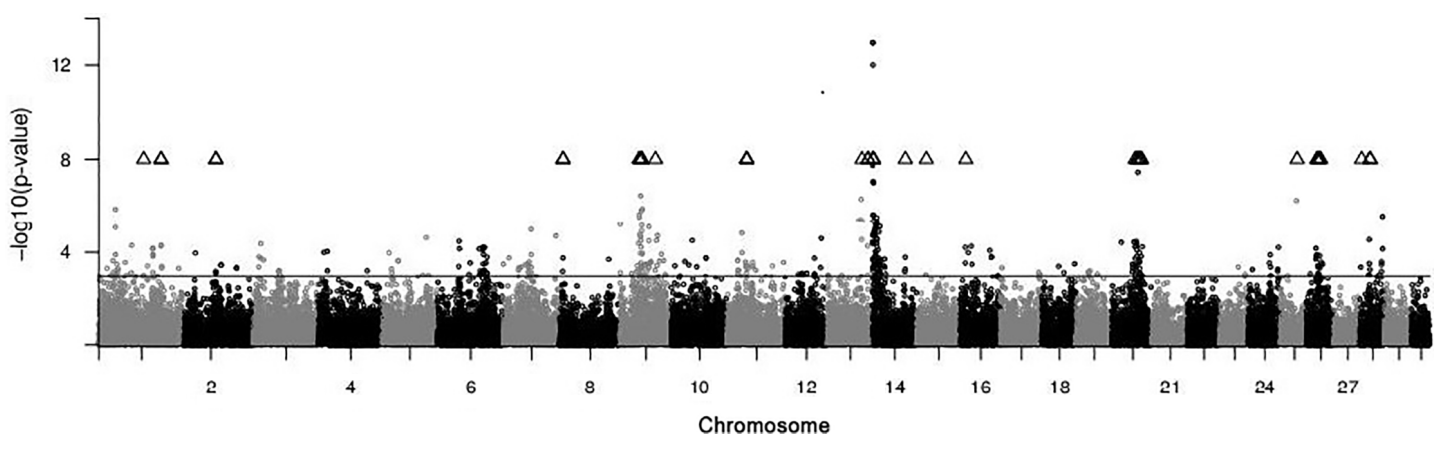

Figure 5. Test statistics of a single-marker GWAS for SNP environmental sensitivity for milk protein yield in a sample of the German Holstein population. The solid line corresponds to a significance level of $P=0.001$. Significant SNPs are indicated by triangles (taken from Streit et al., 2013a).

\section{Mapping populations}

\subsection{Segregating populations}

In contrast to QTL linkage mapping, for GWASs no experimental population (e.g., half-sib or F2 design) has to be established because genome-wide LD is assumed and used for mapping purposes (in linkage mapping the LD is generated within families by the mating design, and this allows for the use of low marker densities). Nevertheless, the study design affects the outcome of a GWAS. A few important aspects will be mentioned in the following. First, the sample size of the experiment affects the power. It is often stated that at least 1000 genotyped and phenotyped individuals have to be included, even for simple traits. This is the minimum number of individuals required for statistical analysis (except for mapping major genes, which are rare for quantitative traits). Larger numbers can be obtained by analyzing several mapping populations jointly, e.g., Holsteins, Jersey and Red cattle breeds, as done by Mao et al. (2016). This leads to a substantially larger mapping population, and the mapping resolution is much higher as well. The latter is because the genetic diversity within the mapping population is much larger (i.e., the hypothetical effective population size is larger, which in turn affects the LD pattern, as described above). It was frequently shown that such across-breed analysis leads to clearer SNP association signals for genes that segregate in all breeds. At the same time, such an approach can be used to validate significant SNP trait associations across breeds. Another validation approach is to use a sample from the same breed, as done by Streit et al. (2013a) (Figs. 2 and 5). Across-breed analyses can be done either by pooling the data and analyzing them jointly or by a meta-analysis, where the results from the within-breed analysis (effect estimates and $p$ values) are combined. The latter is more convenient to apply because each breed has its own fixed and random explanatory variables to be included in the GWAS models.
The density of the SNP panel is an additional important driver for the success of a GWAS experiment. From the expectation of the LD (shown above), it becomes obvious that higher densities are needed for populations with larger effective population sizes. For cattle, besides the standard chip (50k), there is a high-density (HD) SNP panel (777k) available. Especially for across-breed GWAS experiments, dense SNP data are beneficial due to the large hypothetical effective population size. For many breeds, influential sires were re-sequenced and these sequences can be used for imputation (Daetwyler et al., 2014). Hence, with the aid of HD-SNP chip data and the sequence information of some key ancestors, the whole-genome sequence variants can be inferred for all individuals within a mapping population. This, in turn, can be used for GWASs. A prerequisite for association mapping is a high LD between the marker and the causative mutation. A paradigm shift takes place when using genome sequence variants because all variants (i.e., SNPs and causative mutations) are included in the data set. Now the challenge is to identify the causative mutations among all polymorphisms and to separate them from SNPs that are solely in LD with the mutation. The success of a GWAS with genome sequence variants depends strongly on the quality of imputation of these variants in the study population. This is not always ensured but will not be reviewed here. Another problem is the level of multiple testing which increases towards several million correlated tests. A Bonferroni correction is too conservative. A possible solution for this problem is to map the QTL using SNP chip data in a first GWAS run applying standard multiple testing corrections (Bonferroni or FDR). In a second step, fine-mapping of the significant regions can be done using imputed genome sequence variants. Since it is assumed that the regions are significant, no additional stringent significance level has to be applied during fine-mapping. 


\subsection{F2 designs}

Many F2 crosses were established during the last decades, especially in pig breeding (Rothschild et al., 2007). Often, the F2 individuals were phenotyped under standardized conditions (e.g., on experimental farms) for traits that are interesting but very hard to measure, like efficiency traits or meat quality traits. Founder breeds were frequently chosen from Asian and from European breeds. Phylogenetic analysis of whole-genome sequence data revealed distinct lineages of these two types of breeds. In addition, F2 crosses within European types of breeds were established. In many cases one commercially used breed was one of the two founder breeds, e.g., the F2 crosses described in Boysen et al. (2010) and Rückert and Bennewitz (2010); both had Piétrain as one founder breed, which is an important sire line breed in Europe. We studied the LD pattern within an F2 cross derived from distantly related founders (i.e., a Meishan $\times$ Piétrain cross) and within a cross derived from closely related founder breeds (i.e., Landrace/Large White $\times$ Piétrain cross), using porcine SNP chip data. The results for SSC 1 are visualized for each of the two crosses in Fig. 1. As shown there, the LD is high and almost did not decrease with increasing marker distances up to $5 \mathrm{Mb}$ in the Meishan $\times$ Piétrain cross, which implies a poor mapping resolution. In contrast, the LD pattern of the Landrace/Large White $\times$ Piétrain cross is similar to the pattern observed within the Piétrain breed (Fig. 1). Consequently, this results in a similar mapping resolution of such F2 designs and their founder breeds.

The question is whether is possible to map and fine-map genes in porcine F2 designs by SNP chip genotyping and GWASs. Ledur et al. (2009) studied the power of GWASs in F2 crosses by means of simulations and compared it to classical linkage analysis mapping. They found an increase in power and a smaller rate of false positive results in F2 crosses with large sample sizes and high marker densities. In order to continue these investigations, we simulated the two types of porcine F2 crosses described above (Schmid et al., 2017). Thereby, we created a situation where the genome sequence variants of all F2 individuals were available. The results showed that existing F2 crosses generated from closely related founder breeds with whole-genome sequence data available for all individuals could be used to map genes that segregate within a founder breed with a high precision. This is due to the high mapping resolution within this type of cross. Such genes are of interest for breeding purposes, e.g., in the genomic selection program established in the Piétrain breed. In contrast, the mapping precision was very poor in the cross derived from distantly related founder breeds, as expected. The results of the simulation study showed that it might be a worthwhile effort to genotype existing F2 crosses derived from closely related founder breeds with dense SNP panels and conduct GWASs in order to make use of the existing information in the $\mathrm{F} 2$ crosses, especially with regard to the special traits that were collected in these individuals.

\section{Post-GWAS analyses}

The final aims of a mapping experiment are to detect the underlying gene and the causative mutation within the gene. On the level of the DNA, the causality of a mutation can be identified (although not formally proved) by collecting pieces of evidence. The following facts strongly support the causality of a mutation (Mackay, 2001; Meuwissen, 2010). (1) If a mutation is included in the statistical model, no further polymorphism in strong LD with this mutation shows a significant effect. (2) The genotype effects can be validated and are similar in size in different populations and show the same algebraic sign. (3) The complete linkage disequilibrium test (CLD test) and (4) the concordance test have positive results. To verify point 2 , one needs multiple populations. Due to the small number of individuals in experimental populations, this requirement is often difficult to fulfill. The CLD test (point 3; Uleberg and Meuwissen, 2011) is based on two analytic steps. First, all SNPs are tested one by one for association and the test statistics are noted. The second step consists of analyzing the difference in the test statistics. The hypothesis is based on the assumption that the causative mutation explains more variation than any SNP, which is in incomplete LD with the mutation. The concordance test (point 4; Ron and Weller, 2007; Weller and Ron, 2011) tests whether the same SNP allele identifies the same QTL allele $(Q$ or $q)$ in multiple families of QTL-heterozygous parents (which are identified by markers, for instance by using multiple marker regression; see Knott, 2005). Proving the causality of a mutation requires functional studies, but this is not the subject of this review.

\section{Concluding remarks}

Mapping trait-associated SNPs and genes underlying the genetic variance of quantitative traits is still a burning issue in livestock genetic research. In future, two developments can be expected. On the one hand, we already observe that the data sets available for GWAS are increasing from day to day, and in the near future, we will be able to use several hundreds of thousands of individuals. This holds true for traits that are widely used in animal breeding and for which large-scale phenotyping is thus implemented in routine data collection. Combined with improved annotated reference genomes and genome sequence databases, it will be possible to infer the whole-genome sequence variants of the individuals. Thus, it can be expected that the number of detected causative variants will increase for these mainstream traits, especially in across-breed analyses (within breeds the LD structure might prohibit the detection of many causative mutations even in large data sets). On the other hand, phenotypic records of genetically simpler traits can be collected in experimental populations by in-depth phenotyping (e.g., metabolic traits or gene expression traits). The detection of causative genes for these traits requires less large data sets, but a high precision 
in data recording and a well-defined experimental structure are needed.

Data availability. No data sets were used in this article.

Competing interests. The authors declare that they have no conflict of interest.

Acknowledgements. This study was supported by a grant from the German Research Foundation (Deutsche Forschungsgemeinschaft, DFG).

Edited by: Steffen Maak

Reviewed by: two anonymous referees

\section{References}

Bennewitz, J., Solberg, T., and Meuwissen, T. H. E.: Genomic breeding value estimation using nonparametric additive regression models, Genet. Sel. Evol., 41, 20, https://doi.org/10.1186/1297-9686-41-20, 2009.

Bennewitz, J., Edel, C., Fries, R., Meuwissen, T. H. E., and Wellmann, R.: Application of a Bayesian dominance model improves power in quantitative trait genome-wide association analysis, Genet. Sel. Evol., 49, 7, https://doi.org/10.1186/s12711017-0284-7, 2017.

Bolormaa, S., Pryce, J. E., Zhang, Y., Reverter, A., Barendse, W., Hayes, B. J., and Goddard, M. E.: Non-additive genetic variation in growth, carcass and fertility traits of beef cattle, Genet. Sel. Evol., 47, 26, https://doi.org/10.1186/s12711-015-0114-8, 2015.

Boysen, T. J., Tetens, J., and Thaller, G.: Detection of a quantitative trait locus for ham weight with polar overdominance near the ortholog of the callipyge locus in an experimental pig F2 population, J. Anim. Sci., 88, 3167-3172, https://doi.org/10.2527/jas.2009-2565, 2010.

Calus, M. P. L., Groen, A. F., and de Jong, G.: Genotype $\times$ Environment Interaction for Protein Yield in Dutch Dairy Cattle as Quantified by Different Models, J. Dairy Sci., 85, 3115-3123, https://doi.org/10.3168/jds.S0022-0302(02)74399-3, 2002.

Carlborg, Ö. and Haley, C. S.: Opinion: Epistasis: too often neglected in complex trait studies?, Nat. Rev. Genet., 5, 618-625, https://doi.org/10.1038/nrg1407, 2004.

Daetwyler, H. D., Capitan, A., Pausch, H., Stothard, P., van Binsbergen R., Brøndum, R. F., Liao, X., Djari, A., Rodriguez, S. C., Grohs, C., Esquerré, D., Bouchez, O., Rossignol, M.-N., Klopp, C., Rocha, D., Fritz, S., Eggen, A., Bowman, P. J., Coote, D., Chamberlain, A. J., Anderson, C., van Tassell, C. P., Hulsegge, I., Goddard, M. E., Guldbrandtsen, B., Lund, M. S., Veerkamp, R. F., Boichard, D. A., Fries, R., and Hayes, B. J.: Wholegenome sequencing of 234 bulls facilitates mapping of monogenic and complex traits in cattle, Nat. Genet., 46, 858-865, https://doi.org/10.1038/ng.3034, 2014.

Dekkers, J. C. M.: Commercial application of marker- and geneassisted selection in livestock?: Strategies and lessons, J. Anim. Sci., 82, E313-E328, 2004.
Erbe, M., Hayes, B. J., Matukumalli, L. K., Goswami, S., Bowman, P. J., Reich, C. M., Mason, B. A., and Goddard M. E.: Improving accuracy of genomic predictions within and between dairy cattle breeds with imputed high-density single nucleotide polymorphism panels, J. Dairy Sci., 95, 4114-4129, https://doi.org/10.3168/jds.2011-5019, 2012.

Ertl, J., Legarra, A., Vitezica, Z. G., Varona, L., Edel, C., Emmerling, R., and Götz, K.-U.: Genomic analysis of dominance effects on milk production and conformation traits in Fleckvieh cattle, Genet. Sel. Evol., 46, 40, https://doi.org/10.1186/1297-9686-4640, 2014.

Falconer, D. S. and Mackay, T. F. C.: Introduction to Quantitative Genetics, 4th Edn., Longman Group Ltd, London, 1996.

Fernando, R., Toosi, A., Wolc, A., Garrick, D., and Dekkers, J. C. M.: Application of Whole-Genome Prediction Methods for Genome-Wide Association Studies: A Bayesian Approach, J. Agric. Biol. Environ. S., 22, 172-193, https://doi.org/10.1007/s13253-017-0277-6, 2017.

Fernando, R. L., Nettleton, D., Southey, B. R., Dekkers, J. C. M., Rothschild, M. F., and Soller, M.: Controlling the Proportion of False Positives in Multiple Dependent Tests, Genetics, 166, 611619, https://doi.org/10.1534/genetics.166.1.611, 2004.

Gianola, D.: Priors in whole-genome regression: The Bayesian alphabet returns, Genetics, 194, 573-596, https://doi.org/10.1534/genetics.113.151753, 2013.

Gianola, D., Fariello, M. I., Naya, H., and Schön, C.-C.: GenomeWide Association Studies with a Genomic Relationship Matrix: A Case Study with Wheat and Arabidopsis, G3, 3, 3241-3256, https://doi.org/10.1534/g3.116.034256, 2016.

Goddard, M. E., Kemper, K. E., MacLeod, I. M., Chamberlain, A. J., and Hayes, B. J.: Genetics of complex traits: prediction of phenotype, identification of causal polymorphisms and genetic architecture, Proc. Biol. Sci., 283, 20160569, https://doi.org/10.1098/rspb.2016.0569, 2016.

Hayes, B. J.: Overview of Statistical Methods for Genome-Wide Association Studies (GWAS), in: Genome-Wide Association Studies and Genomic Prediction, edited by: Gondro, C., van der Werft, J., and Hayes, B. J., Springer Protocols, New York, 149169, 2013.

Hayes, B. J., Carrick, M., Bowman, P. J., and Goddard, M. E.: Genotype $\times$ Environment Interaction for Milk Production of Daughters of Australian Dairy Sires from Test-Day Records, J. Dairy Sci., 86, 3736-3744, https://doi.org/10.3168/jds.S00220302(03)73980-0, 2003.

Hayes, B. J., Bowman, P. J., Chamberlain, A. J., Savin, K. W., van Tassell, C. P., Sonstegard, T. S., and Goddard, M. E.: A validated genome wide association study to breed cattle adapted to an environment altered by climate change, PLoS One, 4, 1-8, https://doi.org/10.1371/journal.pone.0006676, 2009.

Hayes, B. J., Daetwyler, H. D., and Goddard, M. E.: Models for Genome $\mathrm{x}$ Environment interaction: Examples in livestock, Crop Sci., 56, 2251-2259, https://doi.org/10.2135/cropsci2015.07.0451, 2016.

Hill, W. G. and Weir, B. S.: Maximum-likelihood estimation of gene location by linkage disequilibrium, Am. J. Hum. Genet., 54, 705714, 1994.

Hill, W. G., Goddard, M. E., and Visscher, P. M.: Data and theory point to mainly additive genetic vari- 
ance for complex traits, PLoS Genet., 4, e1000008, https://doi.org/10.1371/journal.pgen.1000008, 2008.

Hu, Y., Rosa, G. J. M., and Gianola, D.: A GWAS assessment of the contribution of genomic imprinting to the variation of body mass index in mice, BMC Genomics, 16, 576, https://doi.org/10.1186/s12864-015-1721-z, 2015.

Knott, S. A.: Regression-based quantitative trait loci mapping: robust, efficient and effective, Philos. T. Roy. Soc. B, 360, 14351442, https://doi.org/10.1098/rstb.2005.1671, 2005.

Ledur, M. C., Navarro, N., and Pérez-Enciso, M.: Large-scale SNP genotyping in crosses between outbred lines: how useful is it?, Heredity, 105, 173-182, https://doi.org/10.1038/hdy.2009.149, 2009.

Lutz, V., Stratz, P., Preuß, S., Tetens, J., Grashorn, M. A., Bessei, W., and Bennewitz, J.: A genome-wide study in a large F2-cross of laying hens reveals novel genomic regions associated with feather pecking and aggressive behavior, Genet. Sel. Evol., 49, 18, https://doi.org/10.1186/s12711-017-0287-4, 2017.

Mackay, T. F. C.: The Genetic Architecture of Quantitative Traits, Annu. Rev. Genet., 35, 303-339, https://doi.org/10.1146/annurev.genet.35.102401.090633, 2001.

MacLeod, I. M., Hayes, B. J., Savin, K. W., Chamberlain, A. J., McPartlan, H. C., and Goddard, M. E.: Power of a genome scan to detect and locate quantitative trait loci in cattle using dense single nucleotide polymorphisms, J. Anim. Breed. Genet., 127, 133142, https://doi.org/10.1111/j.1439-0388.2009.00831.x, 2010.

Mantey, C., Brockmann, G. A., Kalm, E., and Reinsch, N.: Mapping and exclusion mapping of genomic imprinting effects in mouse F 2 families, J. Hered., 96, 329-338, https://doi.org/10.1093/jhered/esi044, 2005.

Mao, X., Sahana, G., De Koning, D.-J., and Guldbrandtsen, B.: Genome-wide association studies of growth traits in three dairy cattle breeds using whole-genome sequence data, J. Anim. Sci., 94, 1426-1437, https://doi.org/10.2527/jas.2015-9838, 2016.

Meuwissen, T. H. E.: Use of whole genome sequence data for QTL mapping and genomic selection, in: Proceedings of the 9th World Congress on Genetics Applied to Livestock Production, Leipzig, Germany, 1-6 August 2010.

Meuwissen, T. H. E., Hayes, B. J., and Goddard, M. E.: Prediction of total genetic value using genome-wide dense marker maps, Genetics, 157, 1819-1829, 2001.

Patterson, N., Price, A. L., and Reich, D.: Population structure and eigenanalysis, PLoS Genet., 2, 2074-2093, https://doi.org/10.1371/journal.pgen.0020190, 2006.

Qanbari, S., Pimentel, E. C. G., Tetens, J., Thaller, G., Lichtner, P., Sharifi, A. R., and Simianer, H.: The pattern of linkage disequilibrium in German Holstein cattle, Anim. Genet., 41, 346-356, https://doi.org/10.1111/j.1365-2052.2009.02011.x,2010.

Ron, M. and Weller, J. I.: From QTL to QTN identification in livestock - Winning by points rather than knock-out: A review, Anim. Genet., 38, 429-439, https://doi.org/10.1111/j.13652052.2007.01640.x, 2007.

Rothschild, M. F., Hu, Z. L., and Jiang, Z.: Advances in QTL mapping in pigs, Int. J. Biol. Sci., 3, 192-197, https://doi.org/10.7150/ijbs.3.192, 2007.

Rückert, C. and Bennewitz, J.: Joint QTL analysis of three connected F2-crosses in pigs, Genet. Sel. Evol., 42, 40, https://doi.org/10.1186/1297-9686-42-40, 2010.
Sahana, G., Guldbrandtsen, B., and Lund, M. S.: Genomewide association study for calving traits in Danish and Swedish Holstein cattle, J. Dairy Sci., 94, 479-486, https://doi.org/10.3168/jds.2010-3381, 2011.

Schmid, M., Wellmann, R., and Bennewitz, J.: Power and precision of QTL mapping in simulated multiple F2 crosses using wholegenome sequence information, submitted, 2017.

Stratz, P., Wimmers, K., Meuwissen, T. H. E., and Bennewitz, J.: Investigations on the pattern of linkage disequilibrium and selection signatures in the genomes of German Piétrain pigs, J. Anim. Breed. Genet., 131, 473-482, https://doi.org/10.1111/jbg.12107, 2014.

Streit, M., Wellmann, R., Reinhardt, F., Thaller, G., Piepho, H. P., and Bennewitz, J.: Using genome-wide association analysis to characterize environmental sensitivity of milk traits in dairy cattle, G3, 3, 1085-1093, https://doi.org/10.1534/g3.113.006536, $2013 a$.

Streit, M., Reinhardt, F., Thaller, G., and Bennewitz, J.: Genomewide association analysis to identify genotype $\times$ environment interaction for milk protein yield and level of somatic cell score as environmental descriptors in German Holsteins, J. Dairy Sci., 96, 7318-7324, https://doi.org/10.3168/jds.2013-7133, 2013 b.

Su, G., Christensen, O. F., Ostersen, T., Henryon, M., and Lund, M. S.: Estimating Additive and Non-Additive Genetic Variances and Predicting Genetic Merits Using Genome-Wide Dense Single Nucleotide Polymorphism Markers, PLoS One, 7, 1-7, https://doi.org/10.1371/journal.pone.0045293, 2012.

Sved, J. A.: Linkage disequilibrium and its expectation in human populations, Twin Res. Hum. Genet., 12, 35-43, https://doi.org/10.1375/twin.12.1.35, 2009.

Tenesa, A., Navarro, P., Hayes, B. J., Duffy, D. L., Clarke, G. M., Goddard, M. E., and Visscher, P. M.: Recent human effective population size estimated from linkage disequilibrium, Genome Res., 2, 520-526, https://doi.org/10.1101/gr.6023607, 2007.

Uleberg, E. and Meuwissen, T. H. E.: The complete linkage disequilibrium test: a test that points to causative mutations underlying quantitative traits, Genet. Sel. Evol., 43, 20, https://doi.org/10.1186/1297-9686-43-20, 2011.

VanRaden, P. M.: Efficient methods to compute genomic predictions, J. Dairy Sci., 91, 4414-4423, https://doi.org/10.3168/jds.2007-0980, 2008.

Verbyla, K. L., Hayes, B. J., Bowman, P. J., and Goddard, M. E.: Accuracy of genomic selection using stochastic search variable selection in Australian Holstein Friesian dairy cattle, Genet. Res., 91, 307-311, https://doi.org/10.1017/S0016672309990243, 2009.

Verbyla, K. L., Bowman, P. J., Hayes, B. J., and Goddard, M. E.: Sensitivity of genomic selection to using different prior distributions, BMC Proc., 4 (Suppl 1):S5, https://doi.org/10.1186/17536561-4-S1-S5, 2010.

Wei, W.-H., Hemani, G., and Haley, C. S.: Detecting epistasis in human complex traits, Nat. Rev. Genet., 15, 722-733, https://doi.org/10.1038/nrg3747, 2014.

Weller, J. I. and Ron, M.: Invited review: Quantitative trait nucleotide determination in the era of genomic selection, J. Dairy Sci., 94, 1082-1090, https://doi.org/10.3168/jds.20103793, 2011. 
Weller, J. I., Kashi, Y., and Soller, M.: Power of Daughter and Granddaughter Designs for Determining Linkage Between Marker Loci and Quantitative Trait Loci in Dairy Cattle, J. Dairy Sci., 73, 2525-2537, 1990.

Wellmann, R. and Bennewitz, J.: The contribution of dominance to the understanding of quantitative genetic variation, Genet. Res., 93, 139-154, https://doi.org/10.1017/S0016672310000649, 2011.
Wellmann, R. and Bennewitz, J.: Bayesian models with dominance effects for genomic evaluation of quantitative traits, Genet. Res, 94, 21-37, https://doi.org/10.1017/S0016672312000018, 2012.

Yang, J., Zaitlen, N. A., Goddard, M. E., Visscher, P. M., and Price, A. L.: Advantages and pitfalls in the application of mixed-model association methods, Nat. Genet., 46, 100-106, https://doi.org/10.1038/ng.2876, 2014. 